\title{
Distribution of Co-Activators CBP and p300 During Mouse Oocyte and Embryo Development
}

\author{
ROLAND P.S. KWOK, ${ }^{1,2,4 *}$ XIAO-TIE LIU, ${ }^{1}$ AND GARY D. SMITH ${ }^{1,3,4 *}$ \\ ${ }^{1}$ Department of Obstetrics and Gynecology, University of Michigan, Ann Arbor, Michigan \\ ${ }^{2}$ Department of Biological Chemistry, University of Michigan, Ann Arbor, Michigan \\ ${ }^{3}$ Department of Physiology, University of Michigan, Ann Arbor, Michigan \\ ${ }^{4}$ Reproductive Science Program, University of Michigan, Ann Arbor, Michigan
}

\begin{abstract}
CAMP response element binding protein (CREB)-binding protein (CBP) and p300 are two structurally related transcriptional co-activators that activate expression of many eukaryotic genes. Current dogma would suggest that these transcriptional coactivators have similar mechanisms of transcription regulation. Studies of CBP or p300 homozygotic mouse mutants indicate that normal embryogenesis requires the existence of both factors. However, whether this is indicative of a dosage effect of these two proteins, or whether these proteins play different roles in mouse embryo development is not clear. Here we demonstrated that both factors are first found in the cytoplasm of oocytes within primordial follicles, and that they enter into the oocyte nucleus at different stages of oocyte growth, suggesting that they may play different roles in gene expression during oocyte growth and development. Consistent with this model, in the pre-implantation mouse embryos, from the two-cell stage to the blastocyst stage, the localizations of CBP and p300 are different, at times opposite, indicating that CBP and p300 also have different functions in early mouse embryogenesis. Mol. Reprod. Dev. 73: 885-894, 2006. () 2006 Wiley-Liss, Inc.
\end{abstract}

Key Words: CREB; CAMP; PKA

\section{INTRODUCTION}

Around the time of birth, mammalian oocytes have finished undergoing mitotic divisions and have entered the dictyate-stage of the first meiotic prophase. These nongrowing oocytes are contained within primordial follicles and remain arrested at this meiotic stage and growth phase until the oocytes and follicles are recruited for development and subsequent growth (Bachvarova, 1985). Nongrowing oocytes within primordial follicles have very little total RNA and are relatively transcriptionally inactive. During follicular development the oocyte growth phase begins with the transition from primordial to primary follicle, continues through secondary and tertiary follicular development and subsides at antrum formation (Bachvarova, 1985). During the growth phase, the oocyte is still arrested in the dictyatestage, which is characterized by very diffuse chromo- somes, and a high rate of transcriptional activity. It has been estimated that during the oocyte growth phase there is approximately a 300 -fold increase in total RNA within the oocyte (Bachvarova, 1985; Wassarman and Kinloch, 1992) of which approximately $10 \%-15 \%$ is mRNA (Bachvarova, 1985). To date the specific transcription regulators involved with gene expression during oogenesis remain unclear.

Transcription continues in the fully-grown oocyte, albeit at a diminished rate in comparison to the growing oocyte, and declines to practically undetectable levels around the time of meiosis resumption (Wassarman and Letourneau, 1976). Following germinal vesicle breakdown (GVB), and during meiotic maturation, as much as $50 \%$ of the mRNA accumulated during oocyte growth is either deadenylated and/or degraded. The remaining transcripts and their translated proteins collectively constitute the maternal products that are required after fertilization to support early embryo development and subsequent gene expression of the zygote (zygotic genome activation, ZGA) (Telford et al., 1990). The major ZGA occurs at the 2-cell stage in mouse embryos (Schultz, 1993). The initiating signal and propagating mechanism involved in ZGA are still unknown; however, post-translational modification of maternally derived proteins appears to be pivotal in regulating gene activation in the early embryo. The importance of protein phosphorylation by cAMP-dependent protein kinase (PKA) has been demonstrated during ZGA (Poueymirou and Schultz, 1989), yet the mechanism remains to be determined.

The most likely mechanism by which PKA could influence ZGA would be through its direct link with transcription factors. Interaction of cAMP with regulatory

Grant sponsor: National Institute of Diabetes \& Digestive \& Kidney Diseases; Grant number: NIH5P60 DK20572.

${ }^{*}$ Correspondence to: Roland P.S. Kwok, and Gary D. Smith, 6428 Medical Science I, 1301 E. Catherine Street, Ann Arbor, MI 48109. E-mail: rkwok@umich.edu; smithgd@umich.edu

Received 11 July 2005; Accepted 10 November 2005

Published online 4 April 2006 in Wiley InterScience (www.interscience.wiley.com).

DOI $10.1002 / \mathrm{mrd} .20440$ 


\section{$886 \quad$ R.P.S. KWOK ET AL.}

subunits of PKA results in release of catalytic subunits that translocate to the nucleus where they initiate a cascade of events leading to transcription of cAMP/PKAresponsive genes. Within the nucleus, the catalytic subunits of PKA phosphorylate and activate CREB, which constitutively binds to the cAMP-response element (CRE) found in the promoter regions of cAMP-responsive genes (Mayr and Montminy, 2001). Recent studies demonstrated that cells require transcriptional co-activators such as CBP or its paralog p300 for efficient transcription in response to cAMP-responsive genes (Goodman and Smolik, 2000). Initially, CBP and p300 were identified by their ability to interact with PKA-phosphorylated and transcriptionally active forms of CREB (Chrivia et al., 1993; Kwok et al., 1994) and the adenoviral protein E1A (Eckner et al., 1994; Lundblad et al., 1995), respectively. It has long been known that the immortalization and generepression functions of 12S E1A depend upon the binding of p300 (Stein et al., 1990; Wang et al., 1993).

CBP and p300 are highly homologous proteins that are well conserved among eukaryotic cells. Homologues of CBP and p300 can be found in mammals (human, mouse, rat) and in organisms such as Drosophila and Caenorhabditis (Giordano and Avantaggiati, 1999; Goodman and Smolik, 2000), but not in yeast or prokaryotes. CBP and p300 belong to the same class of proteins, derived from gene amplification (Pandey et al., 2002). Because of the high degree of sequence similarity between these proteins ( $61 \%$ identical at the amino acid level, and up to $95 \%$ similarity in functional domains), CBP and p300 have been considered to be functionally equivalent (Giordano and Avantaggiati, 1999). They have been shown to interact with several cellular protooncogenes as well as a wide variety of other transcription factors, supporting the notion that $\mathrm{CBP} / \mathrm{p} 300$ plays an important role in controlling cellular functions, including proliferation and transformation. Indeed, mutations in the $C B P$ gene in humans have been linked to Rubinstein-Taybi syndrome (Petrij et al., 1995), a developmental disorder characterized by mental retardation, digital abnormalities, and an increased incidence of certain types of tumors.

Precisely how $\mathrm{CBP} / \mathrm{p} 300$ causes gene activation has not been resolved. One possibility is that $\mathrm{CBP} / \mathrm{p} 300$ activates gene transcription by linking transcriptional activators to the general transcription machinery, such as TFIIB (Kwok et al., 1994), or TFIID (Bisotto et al., 1996; Swope et al., 1996). Consistent with this model, $\mathrm{CBP} / \mathrm{p} 300$ has been demonstrated to be part of the RNA polymerase holoenzyme complex (Kee et al., 1996). Moreover, recent studies have also shown that CBP/ p300 (Ogryzko et al., 1996) and its associated protein P/ CAF (Yang et al., 1996) are histone acetyltransferases, suggesting that $\mathrm{CBP} / \mathrm{p} 300$ may activate gene transcription by remodeling chromatin.

Studies have shown that homozygous CBP or p300 knockout in mice is embryonically lethal, which occurs between E9 and E11.5 (Yao et al., 1998; Kung et al., 2000 ), suggesting that these two factors may play a role in embryonic development. The phenotypes of embryo- nic lethality of CBP or p300 knock out in mice are similar, having defects in neural tube closure, cell proliferation, and cardiac development, suggesting either (1) that the function of CBP and p300 in mouse embryonic development is similar but the presence of both factors is required for proper embryonic development (a dosage effect) or (2) that CBP and p300 play different roles in embryonic development and neither CBP or p300 can compensate for the absence of the other.

To distinguish these two models, we reasoned that if CBP and p300 function similarly in embryonic development, one would expect that their localizations within developing oocytes and early embryos be similar; however, if their localizations were different, it would suggest that they had different functions. In this study, we have studied the distribution of CBP and p300 during oocyte and embryo development from the primordial follicle stage to pre-implantation embryo. Our results indicate that CBP and p300 are differentially regulated during oocyte and preimplantation embryo development. This is the first report of differential localization of these two transcriptional co-activators within the same cell or group of cells during developmental transitions. These data indicate that CBP and p300 may have different functions in mouse oogenesis and early embryogenesis.

\section{MATERIALS AND METHODS Collection of Mouse Ovaries, Oocytes and Embryos}

Ovaries were obtained from CF-1 female mice on day 11 , or 15 (day $0=$ day of birth) without hormonal stimulation or from adults primed with equine chorionic gonadotropin (eCG). For immunohistochemical studies, ovaries were fixed in 4\% paraformaldehyde (prepared fresh daily), rinsed in PBS and embedded in paraffin. Fully-grown GVB-competent oocytes were isolated by manual rupturing of antral follicles from eCG-primed adult ovaries. Oocytes were isolated into modified Human Tubal Fluid media (HTF-HEPES $+0.3 \%$ polyvinylpyrrolidone; Irvine Scientific, Santa Ana, CA; Sigma Chemical Co., St. Louis, MO) containing $0.2 \mathrm{mM}$ isobutylmethylxanthine (IBMX). Germinal vesicle-intact (GVI) oocytes were completely freed of attached cumulus cells in modified HTF containing IBMX by manual pipetting with a hand-pulled borosillicate pipette. Cumulus-free fully grown GVI oocytes were frozen in liquid nitrogen and stored at $-80^{\circ} \mathrm{C}$ until protein extracts were prepared. Some oocytes were cultured in $\mathrm{HTF}+0.3 \%$ bovine serum albumin (BSA) for in vitro maturation to the metaphase I (MI) and MII stage. In addition, pronuclear zygotes were isolated from the oviduct of $\mathrm{B} 3 \mathrm{C} 6 \mathrm{~F} 1$ female mice, the cumulus cells removed, and cultured in $\mathrm{HTF}+0.3 \%$ BSA for 24 , 72 , or $96 \mathrm{hr}$ to the 2-cell, morula, and expanded blastocyst stages, respectively. These oocytes and embryos were subsequently used for immunocytochemical analysis as described below. 


\section{Western Blot Analysis}

Frozen cumulus cell-free, fully grown GVI oocytes were thawed in triple detergent lysis buffer $(50 \mathrm{mM}$ Tris- $\mathrm{HC} 1$ ( $\mathrm{pH}=8.0$ ), $150 \mathrm{mM} \mathrm{NaC} 1,0.02 \% \mathrm{NaN}_{3}, 0.1 \%$ SDS, $0.6 \mathrm{mM} \mathrm{PMSF}, 1 \mu \mathrm{g} / \mathrm{ml}$ aprotinin, leupeptin, and $N$-tosyl-L-phenylalanine chloromethy ketone, $1.0 \%$ NP-40; $0.5 \%$ sodium deoxycholate), vortexed and placed on ice for $15 \mathrm{~min}$. Following centrifugation at $12,000 \mathrm{~g}$, at $4^{\circ} \mathrm{C}$ for $10 \mathrm{~min}$, total protein from 250 mouse oocytes were separated by SDS-PAGE (Laemmli, 1970) and transfered to a PDVF membrane. Blots were incubated with either anti-CBP (Santa Cruz C-22, Santa Cruz, Santa Cruz, CA) or anti-p300 (Santa Cruz N-15) antibodies in TBS plus 0.1\% BSA for $1 \mathrm{hr}$. To control for the specificity of these antibodies, in some studies, antibodies were first incubated with peptides that were used to raise these antibodies before they were used. Enhanced Chemiluminescence Plus (Amersham, Arlington Heights, IL) was used to detect CBP and p300 according to the procedure described by the manufacturer.

\section{Immunohistochemistry}

Fixed, paraffin-embedded ovaries were sectioned at 5 -micron intervals and placed on superfrost-plus slides (Fisher Scientific; Itasca, IL), deparaffinized, placed in $100 \mathrm{mM}$ Glycine Buffer ( $\mathrm{pH}$ 3.63), and microwaved for $10 \mathrm{~min}$ for antigen retrieval (Shi et al., 1995). To reduce the background signal, samples were placed in Trisbuffered saline (TBS; $50 \mathrm{mM}$ Tris $\mathrm{pH} 7.6,150 \mathrm{mM}$ $\mathrm{NaC} 1$ ) containing $5.0 \%$ dimethyl sulfoxide and $0.2 \%$ Tween-20 for $10 \mathrm{~min}$, rinsed in TBS, and incubated for $10 \mathrm{~min}$ in TBS containing $0.3 \% \mathrm{BSA}, 1 \mathrm{mg} / \mathrm{ml}$ sodium azide, and 1.6\% normal goat serum (Vector Laboratories, Burlingame, CA). In addition, endogenous avidin and biotin were neutralized with an avidin/ biotin blocking kit (Vector Laboratories). Slides were rinsed and incubated overnight with anti-CBP or anti-p300 antibodies at room temperature in a humidified chamber; slides were then rinsed, washed (TBS $+0.05 \%$ Tween-20), rinsed again, and incubated with the biotinylated secondary antibody for $30 \mathrm{~min}$ at room temperature. After secondary antibody exposure, slides were quenched in $3.0 \% \mathrm{H}_{2} \mathrm{O}_{2}$ (in $90 \%$ methanol) for $30 \mathrm{~min}$, then rinsed and incubated for $30 \mathrm{~min}$ in avidin-biotin conjugated to peroxidase (Vector Laboratories). After several rinses, sections were exposed to $0.025 \% 3,3^{\prime}$-diaminobenzidine (DAB, Dojindo Labs-Wako Chemical, Richmond, VA), rinsed, and every other section was counterstained with Mayer's hematoxylin (Sigma), dehydrated by three changes of ethanol, then three changes of xylene, and mounted. Mouse hearts were used as positive controls. Negative controls included: (1) elimination of the primary antibody (not shown) and (2) nonimmune rabbit serum in place of the primary antibody.

Ratios of nuclear to cytoplasmic staining intensities for CBP and p300 in relation to oocyte size were quantified using a Leica DMR, image acquisition with a SPOT-RT digital camera (Diagnos) and computerized densitometry (NIH Image, National Information Service, Springfield, VA). Briefly, ovarian section micrographs without hematoxylin staining were digitally converted to grayscale (Adobe Photoshop, Adobe Systems, Inc., Mountain View, CA). Regions of interest containing $80 \%-90 \%$ of either cytoplasm or nucleus were used to calculate mean pixel intensity of CBP or p300 staining within the cytoplasm and nucleus. Differences in nuclear to cytoplasmic staining intensity ratios of CBP compared to p300 were evaluated for statistical significance using Student's unpaired $t$-test. Differences were considered statistically significant at $P<0.05$.

\section{Immunocytochemistry (ICC) and Confocal Microscope Analysis}

In order to identify localization of CBP and p300 during oocyte maturation and preimplantation embryo development, oocytes at the GVI, MI, and MII stage, and embryos were collected and fixed in $2 \%$ paraformaldehyde with $0.04 \%$ Triton X-100. Oocytes and embryos were then blocked overnight with $0.3 \%$ BSA in PBS at $4^{\circ} \mathrm{C}$, and incubated with rabbit polyclonal anti-CBP or anti-p300 antibodies for $1 \mathrm{hr}$ at $37^{\circ} \mathrm{C}$. After washing with $0.3 \%$ BSA plus $0.1 \%$ Tween-20 in PBS, cells were incubated in the same wash buffer for $90 \mathrm{~min}$ at $37^{\circ} \mathrm{C}$. Samples were then reacted with an anti-rabbit Alexa 58conjugated secondary antibody (Molecular Probes, Eugene, OR) diluted to $2 \mu \mathrm{g} / \mathrm{ml}$ for $1 \mathrm{hr}$ at $37^{\circ} \mathrm{C}$. Following complete washing oocyte DNA was labeled with Hoechst for $30 \mathrm{~min}$, washed, and mounted on slides for fluorescence microscopic visualization. Fluorescence microscopic visualization was performed following washing and mounting. All samples were visualized with a Bio-Rad MRC-600 confocal scanning laser microscope.

\section{RESULTS}

\section{Oocyte CBP and p300 Protein Expression and Intra-Cellular Location}

Using antibodies that recognize CBP and p300 (Fig. 1A), CBP (Fig. 1B), or p300 (Fig. 1C), in a western analysis, we have shown that both CBP and p300 are present in fully grown meiotic competent mouse oocytes (Fig. 1). The specificity of the antibodies were confirmed using purified recombinant CBP or p300 proteins expressed and purified from Sf9 cells. These CBP or p300 specific antibodies were subsequently used in the immunohistochemistry studies described below.

To investigate the intracellular localization of CBP and p300, we employed the CBP- or p300-specific antibodies described in Figure 1 in immunohistochemistry studies. Ovarian tissues from eCG-stimulated adult mice contained follicles in all stages of development; primordial, primary, secondary (collectively termed pre-antral), and antral follicles. While oocytes contained in primordial follicles contained CBP and p300, these proteins were predominantly localized to the 


\section{R.P.S. KWOK ET AL.}

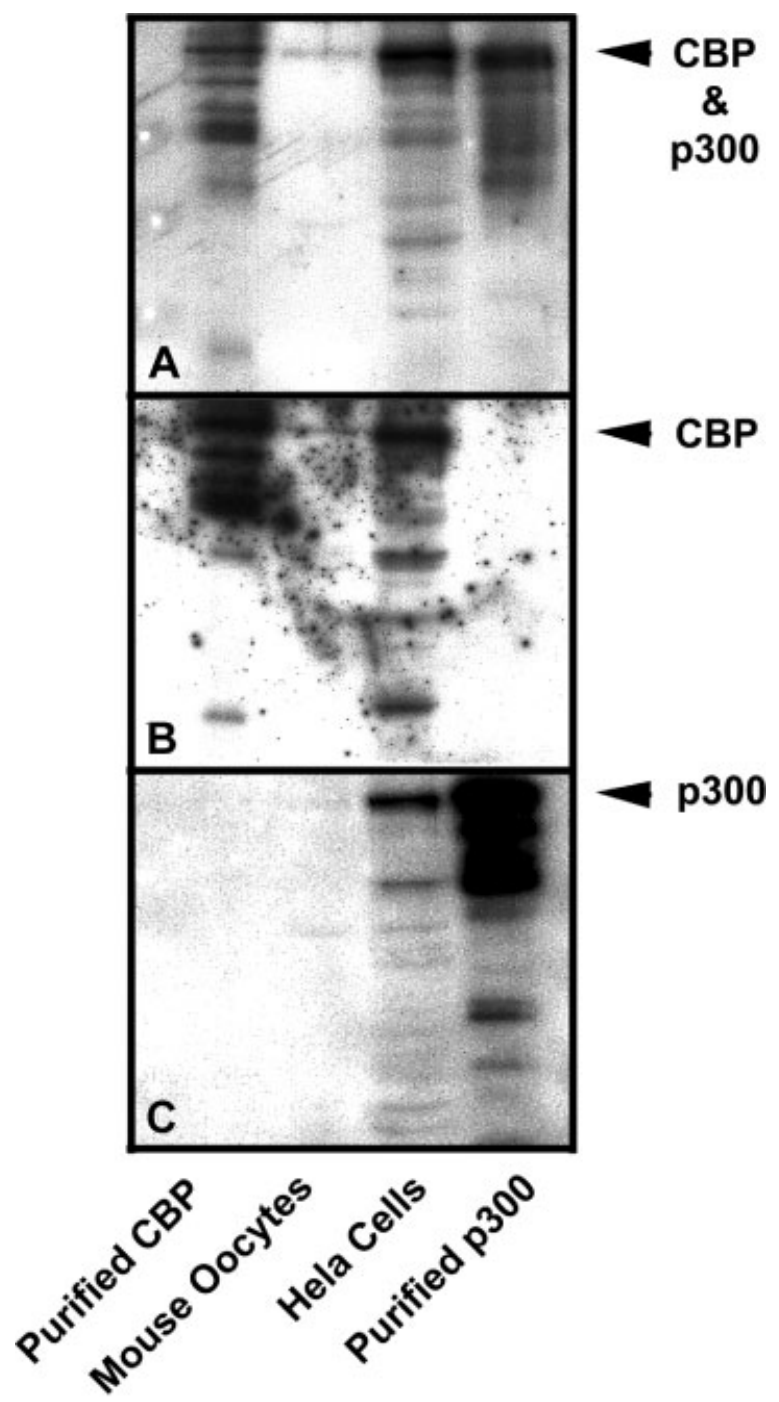

Fig. 1. CBP and p300 are present in mouse oocytes. Purified full length CBP $(0.1 \mu \mathrm{g})$, purified full length p300, HeLa cell extracts $(25 \mu \mathrm{g})$, or extracts from 250 mouse oocytes were separated on an $8 \%$ SDS-PAGE. Western Blot was performed using CBP/p300 antibodies (upper panel), CBP-specific antibodies (middle panel), or p300-specific antibodies (lower panel) as indicated. The signal was detected by ECL plus.

cytoplasm, with very little within the nucleus (see Figs. 3 and 4). However, as pre-antral follicles became developed (primary and secondary), and oocytes began to grow, both CBP and p300 proteins became predominantly localized in the nucleus, with very little protein within the cytoplasm (Fig. 2, panels A-D). CBP and p300 were identified in fully grown meiotic-competent oocytes contained in antral follicles and were present in both the cytoplasm and nucleus, being predominantly in the nucleus, and interestingly in a peri-nucleoli rimming pattern (Fig. 2, panels $\mathrm{E}$ and $\mathrm{F}$ ). Within fully grown oocytes in antral follicles chromatin encircles the nucleolus and has been suggested to be an indicator of meiotic and embryonic developmental competence. These immuno-stainings are specific for CBP or p300 because CBP or p300 antibodies will not cross-react with

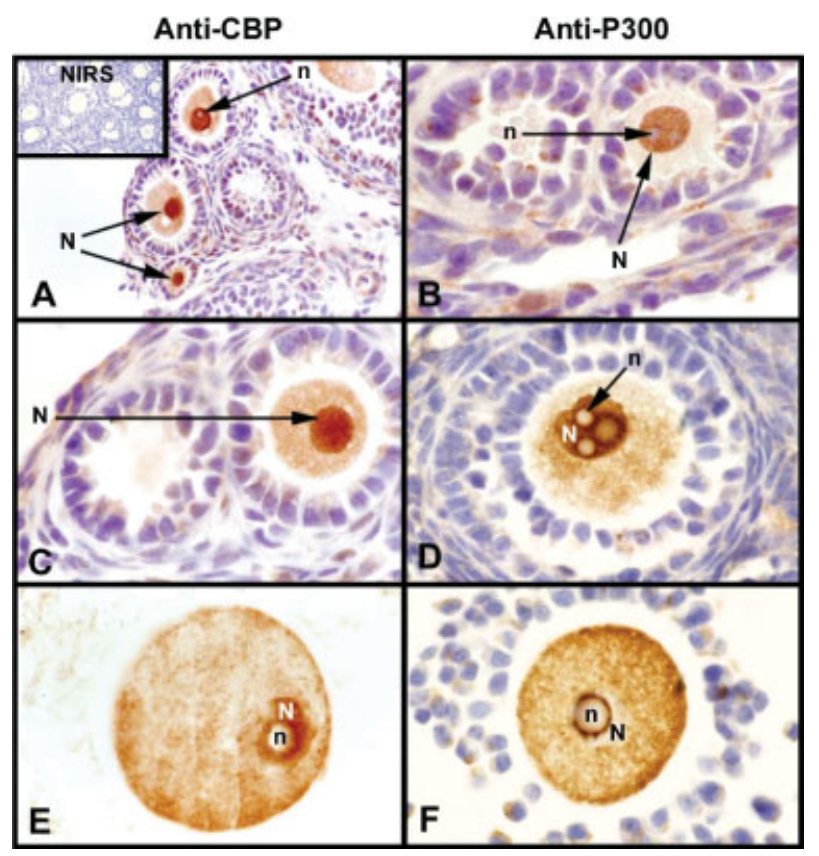

Fig. 2. Immunolocalization of $\mathrm{CBP}(\mathbf{A}, \mathbf{C}, \mathbf{E})$ and $\mathrm{p} 300(\mathbf{B}, \mathbf{D}, \mathbf{F})$ in oocyles of PMSG-stimulated adult mice. $\mathrm{E}$ and $\mathrm{F}$ are oocytes within large antral follicles. N, nucleus; n, nucleolus.

each other as shown in Figure 1. Furthermore, incubating these antibodies with the peptide that was used to raise these antibodies eliminates CBP or p300 immunostaining (data not shown).

\section{Intra-Oocyte Trafficking of CBP and p300 During Oocyte Growth}

Considering the observed change in intra-oocyte CBP and p300 from a cytoplasmic to a nuclear location during initiation of oocyte and follicular growth in adult ovaries, further experiments were conducted in immature mouse ovaries to more closely follow this intraoocyte trafficking of transcriptional co-activators in relation to oocyte growth. Ovaries of 11-day-old mice contain predominantly primordial follicles, some follicles that have initiated growth and developed into primary follicles and a few secondary follicles (Figs. 3 and 4). Immunohistochemistry studies were conducted with nonimmune rabbit serum (specific antibody signal control; Figs. 3A and 4A), anti-CBP (Fig. 3), or anti-p300 (Fig. 4) antibodies with (Figs. 3 and 4, panels A, C, E) and without (Figs. 3 and 4, panels B, D, F) counterstaining of nuclei. Similar to results in adult ovarian tissue, oocytes contained both CBP and p300. In nongrowing oocytes within primordial follicles CBP and p300 were predominantly cytoplasmic (Figs. 3 and 4, panels $\mathrm{C}$ and $\mathrm{D}$ ). Growing oocytes contained in primary or secondary follicles displayed predominant by nuclear CBP and p300 staining in relation to cytoplasmic staining (Figs. 3 and 4, panels $\mathrm{E}$ and $\mathrm{F}$ ).

By eliminating the use of nuclei counterstain, one can convert specific CBP or p300 staining to grey-scale, 


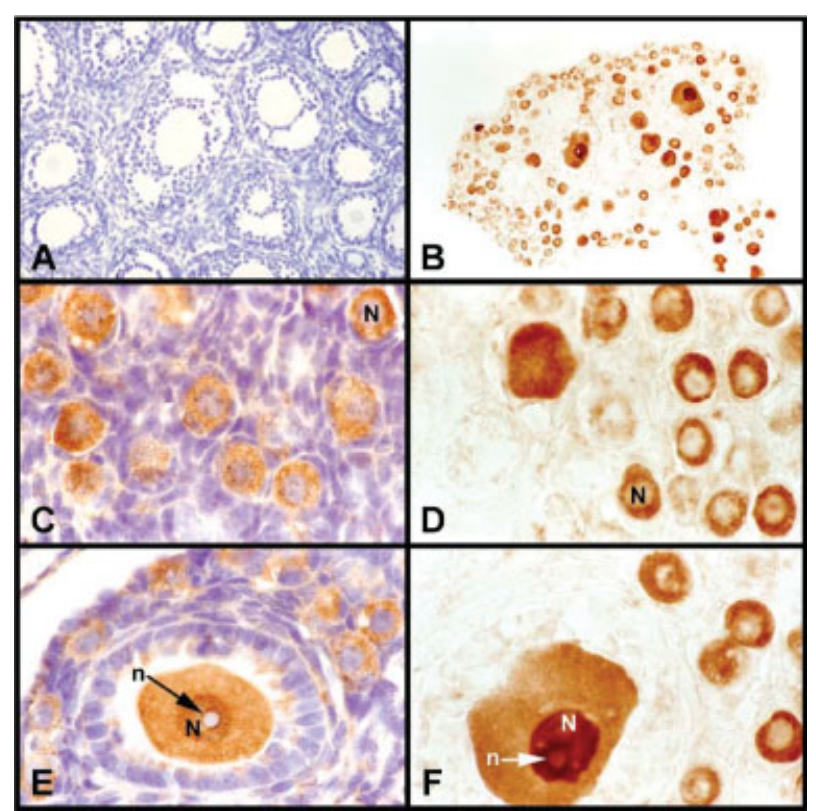

Fig. 3. Immunolocalization of CBP in oocytes of 11-day-old mice with $(\mathbf{A}, \mathbf{C}, \mathbf{E})$ and without $(\mathbf{B}, \mathbf{D}, \mathbf{F})$ hematoxylin counterstain. N-nucleus; n-nucleolus.

encircle the cellular area of interest, and calculate staining intensity. In addition, oocyte diameter can be calculated for each cell assessed for staining intensity. This method allows a 'record of specific nuclear', cytoplasmic, and nuclear/cytoplasmic CBP (Fig. 5) and p300 (Fig. 6) staining intensity in relation to oocyte growth. It also provides a means of quantifying

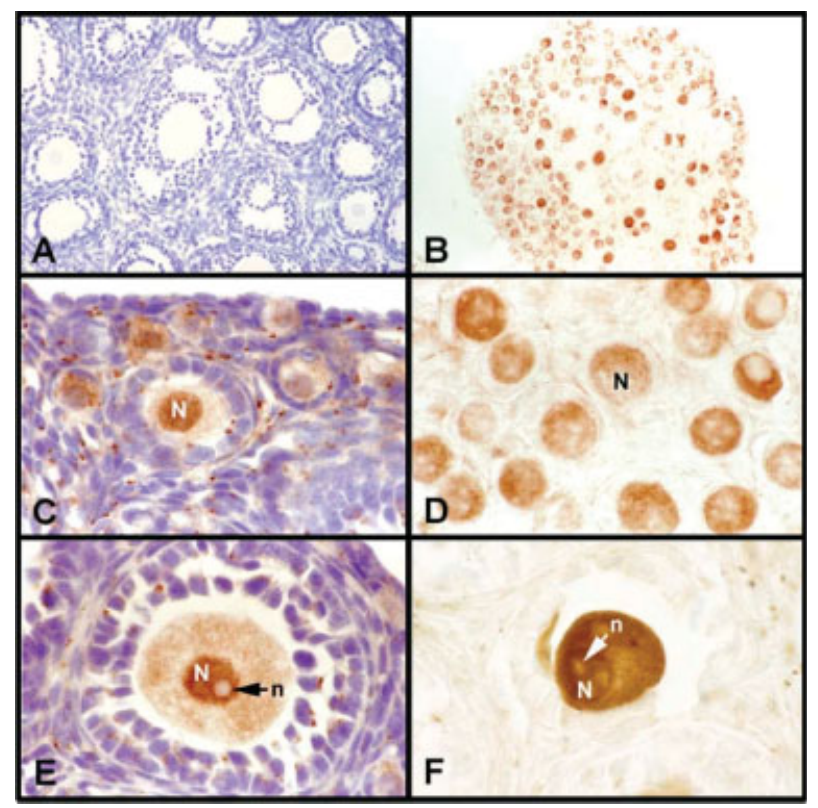

Fig. 4. Immunolocalization of p300 in oocytes of 11-day-old mice with $(\mathbf{A}, \mathbf{C}, \mathbf{E})$ and without $(\mathbf{B}, \mathbf{D}, \mathbf{F})$ hematoxylin counterstain. $\mathrm{N}$-nucleus; n-nucleolus.

\section{D11 CBP Staining}

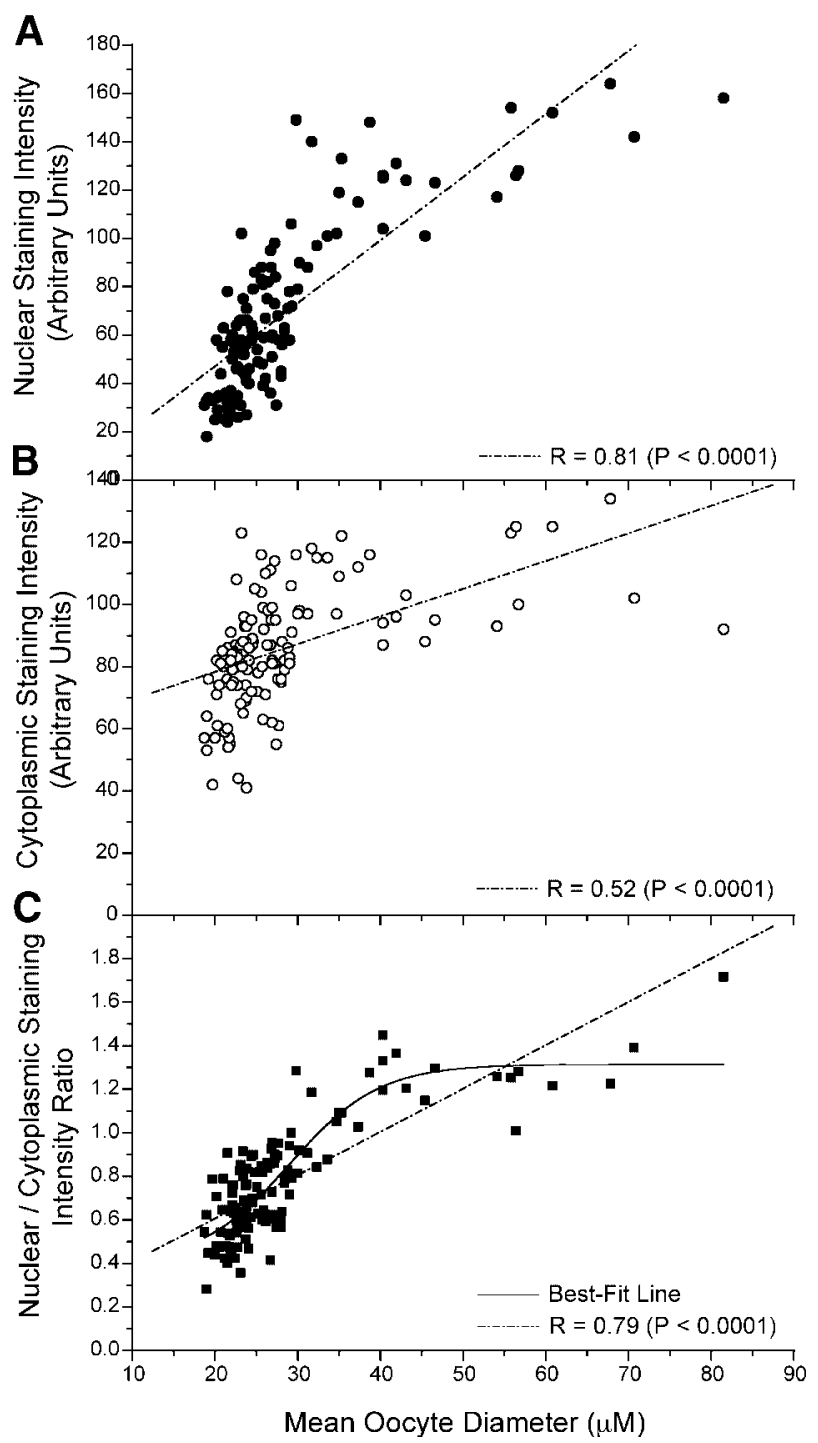

Fig. 5. Nuclear (A), cytoplasmic (B), and nuclear/cytoplasmic ratio (C) staining intensity of CBP in oocytes of differing sizes contained in ovaries of 11-day-old mice. Each symbol represents a single point of measure. Methods and statistical analyses are discussed in detail within the text.

intracellular trafficking patterns in relation to oocyte development and growth. Nuclear CBP and p300 staining intensity was low in nongrowing oocytes. When oocyte began to grow, CBP and p300 trafficked to the nucleus where staining intensity significantly increased $[\mathrm{CBP}$, regression coefficient $(\mathrm{R})=0.81 ; \mathrm{p} 300, \mathrm{R}=0.58$; both $P<0.001]$. Interestingly, while cytoplasmic staining intensity of CBP was positively correlated with oocyte growth $(\mathrm{R}=0.52 ; P<0.001)$ the cytoplasmic staining intensity of p300 did not significantly change in relation to oocyte growth $(\mathrm{R}=0.22 ; P>0.05)$. Lastly, both CBP and p300 nuclear/cytoplasmic staining intensity ratios were significantly correlated, in a positive manner, with oocyte growth $(\mathrm{CBP}, \mathrm{R}=0.79 ; \mathrm{p} 300$, 


\section{D11 p300 Staining}

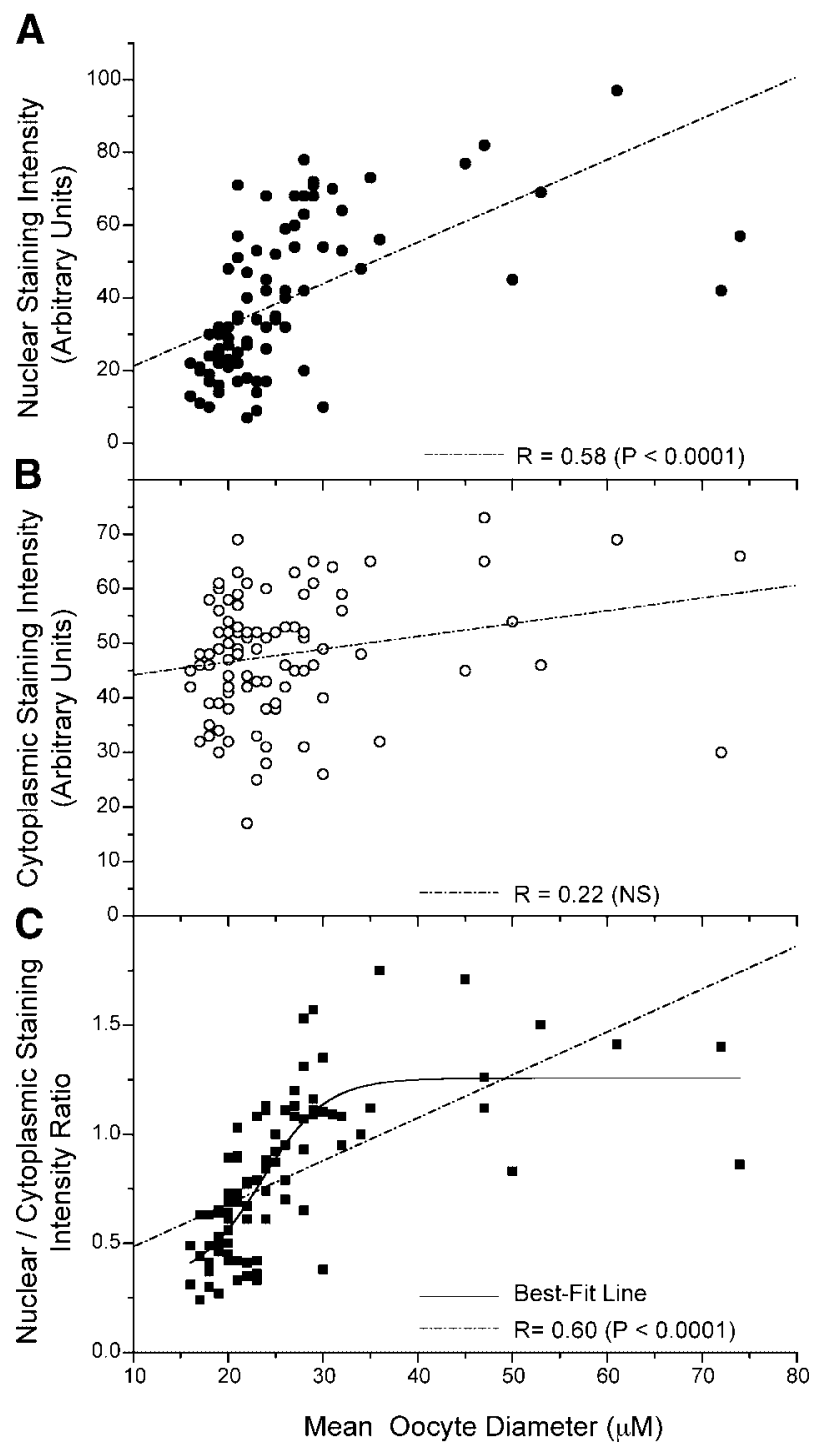

Fig. 6. Nuclear (A), cytoplasmic (B), and nuclear/cytoplasmic ratio (C) staining intensity of p300 in oocytes of differing sizes contained in ovaries of 11-day-old mice. Each symbol represents a single point of measure. Methods and statistical analyses are discussed in detail within the text.

$\mathrm{R}=0.60$; both $P<0.001$ ). When a best-fit line was applied to the CBP and p300 nuclear/cytoplasmic staining intensity plots it was observed that p300 plateaued at greater than 1 (equal nuclear and cytoplasmic staining intensity) at a smaller oocyte size $(\sim 30 \mu \mathrm{m})$ than $\mathrm{CBP}(\sim 40 \mu \mathrm{m})$. These results indicate that, on average, trafficking of p300 into the nucleus occurs prior to trafficking of CBP.

\section{Intra-Oocyte Trafficking and Structural} Associations of CBP and p300 During Meiosis

With both CBP and p300 present in the nuclei of fully grown meiotic-competent GV-intact oocytes, the next obvious question relates to intra-oocyte localization during the actual event of meiosis. Using immunocytochemistry, chromatin labeling, and confocal scanning laser microscopy, it was confirmed that CBP and p300 are predominantly nuclear in GV-intact oocytes (Figs. 7 and 8). Following GVB, CBP is released into the cytoplasm where it evenly distributes, except in close proximity to condensing metaphase chromatin. In both MI and MII oocytes, CBP is markedly absent in regions associated with condensed chromatin (Fig. 7). In contrast, upon GVB at both MI and MII, p300 from within the nucleus and cytoplasm appears to coalesce in close association with condensed chromatin (Fig. 8). This differing intracellular location of CBP and p300 during meiosis is striking considering that these two transcriptional co-activators that have been previously considered to be functionally equivalent.
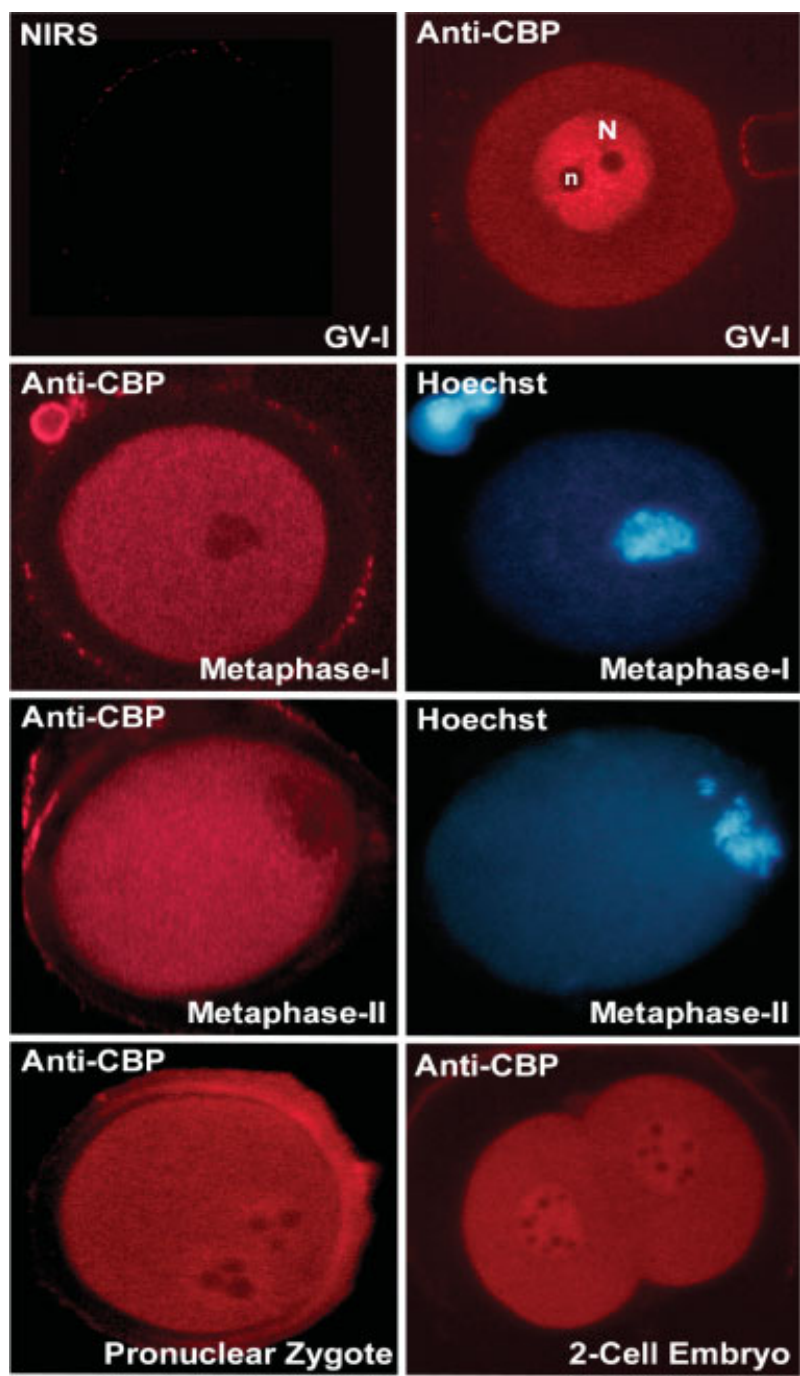

Fig. 7. CBP identification and translocation during resumption of meiosis. NIRS, nonimmune rabbit serum; red stain, CBP; blue stain (Hoechst), chromatin. Note: antibodies and Hoechst staining of the same oocyte within each meiotic stage. 

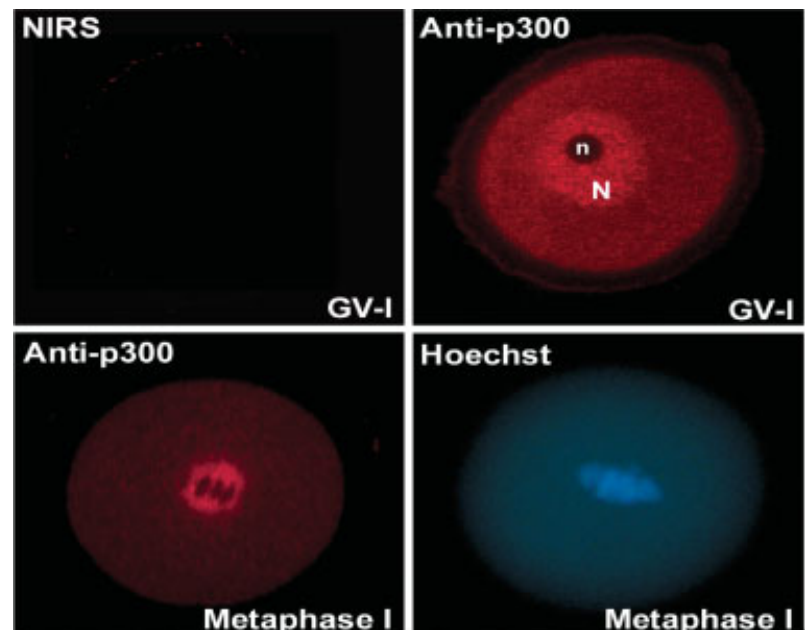

Hoechst
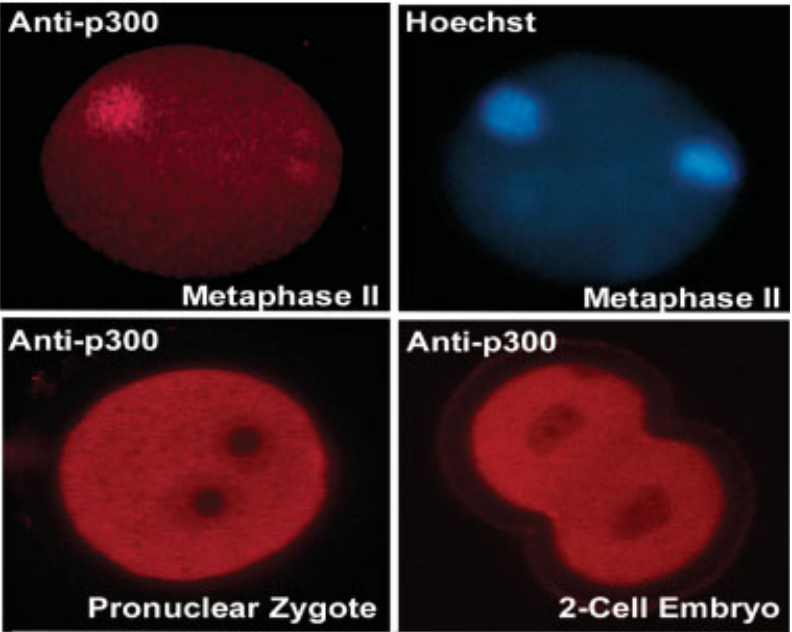

Fig. 8. p300 identification and translocation during resumption of meiosis. NIRS, nonimmune rabbit serum; red stain, p300; blue stain (Hoechst), chromatin. Note: antibodies and Hoechst staining of the same oocyte within each meiotic stage.

\section{Differential CBP and p300 Intracellular Locations During Embryo Development}

Many proteins are carried over from the oocyte to the embryo and provide critical regulatory functions during early preimplantation embryo development. Such might be the case for transcriptional co-activators, CBP and p300. Upon fertilization, yet before ZGA, CBP is both cytoplasmic and nuclear. However, whereas CBP was not associated with chromatin during meiosis, it does relocate to the nuclei in pronuclear stage zygotes (Fig. 7). p300 again shows divergence from CBP in regard to intracellular location following fertilization. While both $\mathrm{CBP}$ and p300 were predominantly nuclear in GV-intact oocytes, p300 did not relocate to the pronuclei, as is the case for CBP. In pronuclear stage zygotes, p300 is predominantly a cytoplasmic protein with a marked reduction in the nucleus (Fig. 8). This predominantly nuclear CBP and cytoplasmic p300 staining pattern is more pronounced at the 2-cell stage when mouse oocytes undergo major ZGA. In the morula and expanded blastocyst, CBP is both nuclear and cytoplasmic, whereas, compared to CBP, p300 remains predominantly cytoplasmic as indicated by the honeycomb-like staining pattern (Fig. 9).

\section{DISCUSSION}

\section{CBP and p300 Act as Coactivators During Oocyte Growth}

The current model of female reproduction proposes that female mammals are born with all the oocytes that they will possess during their life-span, and these oocytes are arrested in prophase of meiosis I. After birth, and until menopause, ovaries contain primordial follicles containing a single layer of squamous granulosa cells and an oocyte, both in a state of growth and developmental quiescence. Cohorts of primordial follicles, and their companion oocytes, are recruited to initiate growth and development. It has been suggested that prior to initiation of oocyte growth, oocyte transcriptional activity is quite low. In contrast, following oocyte growth-initiation, and until growth completion around the time of follicle antrum formation, the oocyte has a high degree of transcriptional activity. How oocyte transcription is regulated during this developmental growth period is poorly understood. Because CBP and p300 are two transcriptional co-activators that have

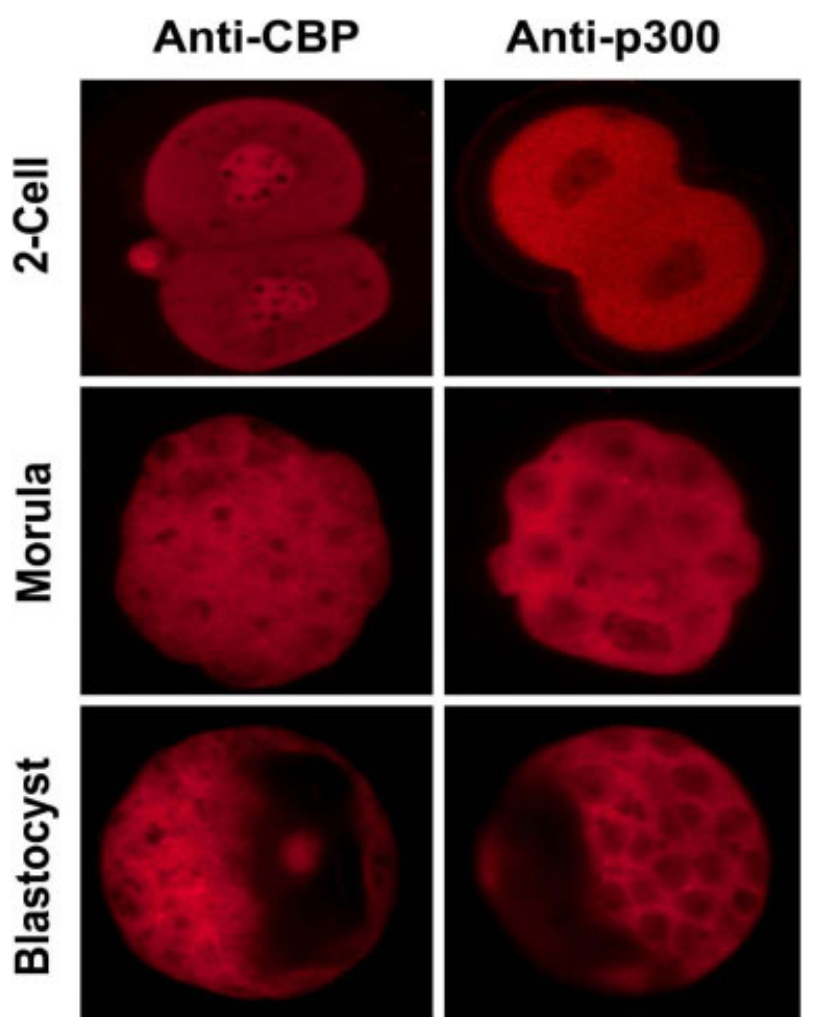

Fig. 9. Identification and intracellular location of CBP and p300 in PN-zygotes, and 2-cell, morula, and blastocyst mouse embryos. Red stain is specific for CBP or p300 depending on which antibodies are used. Note: the lack of CBP and p300 staining in nucleolus; intense CBP in nucleus; reduced p300 in nucleus. [See color version online at www.interscience.wiley.com.] 


\section{$892 \quad$ R.P.S. KWOK ET AL.}

been shown to be involved in integrating numerous signals in gene expression, our studies focused on the timing as well as the location of their expression in oocyte development.

It is widely established that CBP and p300 expression are regulated during mouse post-implantation embryogenesis (Yao et al., 1998; Kato et al., 1999; Shikama et al., 2003) and that deregulation of their synthesis leads to detrimental consequences. But, whether CBP and p300 play a role in oocyte growth and preimplantation embryo is not known. In our studies, using immunocytochemical staining techniques, we have shown that both CBP and p300 are present in mouse oocytes in various stages of development. We have confirmed the specificity of these antibodies in a Western Blot analysis showing that they do not crossreact with each other (Fig. 1). Furthermore, preincubation with peptides that were used to raise these antibodies eliminates CBP and p300 immunostaining (data not shown). Interestingly, both proteins are found in the cytoplasm of oocytes contained in primordial follicles, but in subsequent stages of oocyte growth and follicular development, oocyte CBP and p300 are found primarily in the nucleus. This pattern of trafficking of CBP and p300 from the cytoplasm to the nucleus corresponds to a developmental time point when oocyte growth rate and gene activation are high. This would suggest that CBP and p300 are required for gene expression during oocyte growth and development. Recently, CBP has been shown to migrate from the nucleus to the cytoplasm after inhibition of deacetylase with Trichostatin A (Cohen et al., 2004). The functional significant of the cytoplasm-to-nucleus trafficking of CBP and p300 is not clear. One possibility is that the timing of CBP and p300 trafficking into the nucleus is regulated, and may correspond with the onset of CBP or p300-dependent functions.

This model suggests that the translocation of CBP and p300 in oocytes from the cytoplasm to the nucleus may be required for oocyte and/or follicle development. Recent studies have shown that two TGF- $\beta$ superfamily proteins, growth differentiation factor-9 (GDF-9), and bone morphogenetic protein-15 (BMP-15), are important in follicular development. Both proteins are found in primary follicles. Interestingly, follicular development in GDF knockout mice is severely compromised (Dong et al., 1996), as are rates of ovulation and fertilization in Bmp15 null mice (Yan et al., 2001). Studies have also shown that CBP and p300 act as coactivators for TGF signaling pathways (Iyer et al., 2004); however, whether CBP and p300 play a role in the expression of these two TGF- $\beta$ proteins remains unclear.

\section{CBP and p300 Play Different Roles During Oocyte Meiosis and Early Embryonic Development}

While the localizations of CPB and p300 are similar in oocytes of preantral and antral follicles, our results indicate that the distribution of these two co-activators are quite different during the actual event of meiosis and during preimplantation mouse embryo development, suggesting functional differences between CBP and p300. Following GVB at both MI and MII, p300 is colocalized with chromatin while CBP is distributed in the cytoplasm. The lack of p300 cytoplasmic distribution during resumption of meiosis and its association with chromatin at MI and MII is striking and interesting. The precise intracellular location of p300 may also have developmental consequences, especially to nuclear transfer and cloning technology. One could envision that removal of MII chromatin during generation of a cytoplast for nuclear transfer has the potential of significantly reducing the $\mathrm{p} 300$ available for subsequent early embryo development, which may inadvertently result in a phenocopy of the 1300 knockout mouse. These findings stress the importance of knowledge of not only expression levels of a specific protein, but also its intracellular location and trafficking patterns during development.

Previous studies have shown that homozygous CBP or p300 knockouts are embryonic lethals, which occurs between E9 and E11.5 (Yao et al., 1998; Kung et al., 2000), and the phenotypes of these knockout mice are similar, having defects in neural tube closure, cell proliferation, and cardiac development. Because CBP and p300 cannot replace each other in the homozygous, some suggest that CBP and p300 play different roles in mouse development. Partanen et al. (1999) have shown different localization of CBP and p300 in heart cells, notochord, and rib cartilage of mouse embryo. Our results are consistent with this model. Our results suggest that in early embryogenesis, $\mathrm{CBP}$ and p300 play distinct roles in cell division. Studies have shown that both CBP and p300 are required in cell-cycle progression (Rajabi et al., 2005), however their precise roles in this process are not clear. To our knowledge, their intracellular distribution during cell-cycle progression have not been reported. We found that CBP is localized mainly within the nucleus, while p300 is localized in the cytoplasm after the two-cell stage suggesting differences in functions. It is not surprising that CBP and p300 cannot replace each other in these processes. It is known that patients suffering from Rubinstein-Taybi syndrome, an autosomal dominant syndrome characterized by abnormal growth and mental retardation, have CBP gene mutations (resulting in lower CBP levels) while the $p 300$ gene of these patients was considered to be normal. Indeed, in mice lacking a single allele of CBP, abnormal skeletal patterning is observed in embryos (Tanaka et al., 1997), which is consistent with the partial depletion of CBP in patients with RubinsteinTaybi syndrome. Interestingly, a recent study has revealed that some of the patients with RubinsteinTaybi syndrome who have no CBP gene mutation have p300 gene mutation (Kishimoto et al., 2005). Furthermore, mutation of p300 was reported to be associated with tumor formation of gastrointestinal origin (Muraoka et al., 1996).

The distinct roles of CBP and p300 are not confined to early embryo development however. For example, in F9 
cells, retinoic acid induced differentiation is dependent on p300 but not CBP. Using hammerhead ribozymes capable of cleaving either p300 or CBP messenger RNAs, F9 cells expressing a p300-specific ribozyme became resistant to retinoic-acid-induced differentiation, whereas cells expressing a CBP-specific ribozyme were unaffected (Ugai et al., 1999). Furthermore, recent studies have shown that mutation of the CREB-binding domain of p300, but not CBP, disrupts the binding of p300 with c-Myb, resulting in a defect in haematopoiesis (Kasper et al., 2002). Conversely, CBP, but not p300, is essential for hematopoietic stem cell self-renewal. p300, but not CBP, however, is crucial for proper hematopoietic differentiation (Rebel et al., 2002).

The mechanism by which CBP and p300 function and localize differently in cells remains unknown. The primary amino acid sequences of CBP and p300 are well conserved (86\%-93\%) within their functional domains, which include three $\mathrm{CH}$ regions, a bromodomain, a CREB-binding domain, and a HAT domain (Giles et al., 1998). To our knowledge, CBP and p300 have identical functions when tested in vitro. Furthermore, recently, we have also found that the nuclear localization signal within CBP and p300 is conserved and is localized at the amino termini of these two proteins (manuscript in preparation). Thus, these findings prompt us to speculate that differences between CBP and p300 function and localization may lie within the unconserved sequences between the known functional domains. Whether these unconserved sequences interact with other factors allowing CBP or p300 to function or localize differently from its counterpart remains to be determined.

In summary, the results presented in this study suggest that CBP and p300 have different functions during mouse oocyte growth and development and early embryogenesis.

\section{ACKNOWLEDGMENTS}

We thank Amanda Hutchins-Wolfbrandt for her help in preparing the figures. RPSK is supported by Michigan Diabetes Research and Training Center funded by NIH5P60 DK20572 from the National Institute of Diabetes \& Digestive \& Kidney Diseases.

\section{REFERENCES}

Bachvarova R. 1985. Gene expression during oogenesis and oocyte development in mammals. Dev Biol (NY) 1:453-524.

Bisotto S, Minorgan S, Rehfuss RP. 1996. Identification and characterization of a novel transcriptional activation domain in the CREBbinding protein. J Biol Chem 271:17746-17750.

Chrivia JC, Kwok RP, Lamb N, Hagiwara M, Montminy MR, Goodman RH. 1993. Phosphorylated CREB binds specifically to the nuclear protein CBP. Nature 365:855-859.

Cohen HY, Lavu S, Bitterman KJ, Hekking B, Imahiyerobo TA, Miller C, Frye R, Ploegh H, Kessler BM, Sinclair DA. 2004. Acetylation of the $\mathrm{C}$ terminus of Ku70 by CBP and PCAF controls Bax-mediated apoptosis. Mol Cell 13:627-638.

Dong J, Albertini DF, Nishimori K, Kumar TR, Lu N, Matzuk MM. 1996. Growth differentiation factor-9 is required during early ovarian folliculogenesis. Nature 383:531-535.
Eckner R, Ewen ME, Newsome D, Gerdes M, DeCaprio JA, Lawrence JB, Livingston DM. 1994. Molecular cloning and functional analysis of the adenovirus E1A-associated $300-\mathrm{kD}$ protein (p300) reveals a protein with properties of a transcriptional adaptor. Genes Dev 8 : 869-884.

Giles RH, Peters DJ, Breuning MH. 1998. Conjunction dysfunction: CBP/p300 in human disease. Trends Genet 14:178-183.

Giordano A, Avantaggiati ML. 1999. p300 and CBP: Partners for life and death. J Cell Physiol 181:218-230.

Goodman RH, Smolik S. 2000. CBP/p300 in cell growth, transformation, and development. Genes Dev 14:1553-1577.

Iyer NG, Ozdag H, Caldas C. 2004. p300/CBP and cancer. Oncogene 23: 4225-4231.

Kasper LH, Boussouar F, Ney PA, Jackson CW, Rehg J, van Deursen JM, Brindle PK. 2002. A transcription-factor-binding surface of coactivator p300 is required for haematopoiesis. Nature 419:738743.

Kato Y, Shi Y, He X. 1999. Neuralization of the Xenopus embryo by inhibition of p300/CREB-binding protein function. J Neurosci 19: 9364-9373.

Kee BL, Arias J, Montminy MR. 1996. Adaptor-mediated recruitment of RNA polymerase II to a signal-dependent activator. J Biol Chem 271:2373-2375.

Kishimoto M, Kohno T, Okudela K, Otsuka A, Sasaki H, Tanabe C, Sakiyama T, Hirama C, Kitabayashi I, Minna JD, Takenoshita S, Yokota J. 2005. Mutations and deletions of the $C B P$ gene in human lung cancer. Clin Cancer Res 11:512-519.

Kung AL, Rebel VI, Bronson RT, Ch'ng LE, Sieff CA, Livingston DM, Yao TP. 2000. Gene dose-dependent control of hematopoiesis and hematologic tumor suppression by CBP. Genes Dev 14:272277 .

Kwok RP, Lundblad JR, Chrivia JC, Richards JP, Bachinger HP, Brennan RG, Roberts SG, Green MR, Goodman RH. 1994. Nuclear protein CBP is a coactivator for the transcription factor CREB [see comments]. Nature 370:223-226.

Lundblad JR, Kwok RP, Laurance ME, Harter ML, Goodman RH. 1995. Adenoviral E1A-associated protein p300 as a functional homologue of the transcriptional co-activator CBP. Nature 374:85-88.

Mayr B, Montminy M. 2001. Transcriptional regulation by the phosphorylation-dependent factor CREB. Nat Rev Mol Cell Biol 2: 599-609.

Muraoka M, Konishi M, Kikuchi-Yanoshita R, Tanaka K, Shitara N, Chong JM, Iwama T, Miyaki M. 1996. p300 gene alterations in colorectal and gastric carcinomas. Oncogene 12:1565-1569.

Ogryzko VV, Schiltz RL, Russanova V, Howard BH, Nakatani Y. 1996. The transcriptional coactivators p300 and CBP are histone acetyltransferases. Cell 87:953-959.

Pandey R, Muller A, Napoli CA, Selinger DA, Pikaard CS, Richards EJ, Bender J, Mount DW, Jorgensen RA. 2002. Analysis of histone acetyltransferase and histone deacetylase families of Arabidopsis thaliana suggests functional diversification of chromatin modification among multicellular eukaryotes. Nucleic Acids Res 30:50365055.

Partanen A, Motoyama J, Hui CC. 1999. Developmentally regulated expression of the transcriptional cofactors/histone acetyltransferases CBP and p300 during mouse embryogenesis. Int J Dev Biol 43: 487-494.

Petrij F, Giles RH, Dauwerse HG, Saris JJ, Hennekam RC, Masuno M, Tommerup N, van Ommen GJ, Goodman RH, Peters DJ, et al. 1995. Rubinstein-Taybi syndrome caused by mutations in the transcriptional co-activator CBP [see comments]. Nature 376:348-351.

Poueymirou WT, Schultz RM. 1989. Regulation of mouse preimplantation development: Inhibition of synthesis of proteins in the two-cell embryo that require transcription by inhibitors of cAMP-dependent protein kinase. Dev Biol 133:588-599.

Rajabi HN, Baluchamy S, Kolli S, Nag A, Srinivas R, Raychaudhuri P, Thimmapaya B. 2005. Effects of depletion of CREB-binding protein on c-Myc regulation and cell cycle $\mathrm{G}_{1}-\mathrm{S}$ transition. J Biol Chem 280 : 361-374.

Rebel VI, Kung AL, Tanner EA, Yang H, Bronson RT, Livingston DM. 2002. Distinct roles for CREB-binding protein and p300 in hematopoietic stem cell self-renewal. Proc Natl Acad Sci USA 99:14789-14794. 


\section{$894 \quad$ R.P.S. KWOK ET AL.}

Schultz RM. 1993. Regulation of zygotic gene activation in the mouse. Bioessays 15:531-538.

Shi SR, Imam SA, Young L, Cote RJ, Taylor CR. 1995. Antigen retrieval immunohistochemistry under the influence of $\mathrm{pH}$ using monoclonal antibodies. J Histochem Cytochem 43:193-201.

Shikama N, Lutz W, Kretzschmar R, Sauter N, Roth JF, Marino S, Wittwer J, Scheidweiler A, Eckner R. 2003. Essential function of p300 acetyltransferase activity in heart, lung and small intestine formation. Embo J 22:5175-5185.

Stein RW, Corrigan M, Yaciuk P, Whelan J, Moran E. 1990. Analysis of E1A-mediated growth regulation functions: Binding of the 300kilodalton cellular product correlates with E1A enhancer repression function and DNA synthesis-inducing activity. J Virol 64:4421-4427.

Swope DL, Mueller CL, Chrivia JC. 1996. CREB-binding protein activates transcription through multiple domains. J Biol Chem 271:28138-28145.

Tanaka Y, Naruse I, Maekawa T, Masuya H, Shiroishi T, Ishii S. 1997. Abnormal skeletal patterning in embryos lacking a single Cbp allele: A partial similarity with Rubinstein-Taybi syndrome. Proc Natl Acad Sci USA 94:10215-10220.

Telford NA, Watson AJ, Schultz GA. 1990. Transition from maternal to embryonic control in early mammalian development: A comparison of several species. Mol Reprod Dev 26:90-100.
Ugai H, Uchida K, Kawasaki H, Yokoyama KK. 1999. The coactivators p300 and CBP have different functions during the differentiation of F9 cells. J Mol Med 77:481-494.

Wang HG, Rikitake Y, Carter MC, Yaciuk P, Abraham SE, Zerler B, Moran E. 1993. Identification of specific adenovirus E1A N-terminal residues critical to the binding of cellular proteins and to the control of cell growth. J Virol 67:476-488.

Wassarman PM, Kinloch RA. 1992. Gene expression during oogenesis in mice. Mutat Res 296:3-15.

Wassarman PM, Letourneau GE. 1976. RNA synthesis in fully-grown mouse oocytes. Nature 261:73-74.

Yan C, Wang P, DeMayo J, DeMayo FJ, Elvin JA, Carino C, Prasad SV, Skinner SS, Dunbar BS, Dube JL, Celeste AJ, Matzuk MM. 2001 Synergistic roles of bone morphogenetic protein 15 and growth differentiation factor 9 in ovarian function. Mol Endocrinol 15:854866.

Yang XJ, Ogryzko VV, Nishikawa J, Howard BH, Nakatani Y. 1996. A p300/CBP-associated factor that competes with the adenoviral oncoprotein E1A. Nature 382:319-324.

Yao TP, Oh SP, Fuchs M, Zhou ND, Ch'ng LE, Newsome D, Bronson RT Li E, Livingston DM, Eckner R. 1998. Gene dosage-dependent embryonic development and proliferation defects in mice lacking the transcriptional integrator p300. Cell 93:361-372. 